school attendance and quality of life. Identifying the child at increased risk of future asthma attacks could inform clinical management and targeting of care. We aimed to systematically review the literature to identify and weight factors associated with increased risk of attacks in children with asthma aged 512 years.

Methods Using Cochrane methodology, we systematically searched six databases and undertook forward and backward citation searches, with no date/language restrictions. Two reviewers independently selected studies for inclusion, assessed methodological quality, and extracted data. An expert panel of four clinicians independently assessed each factor for both magnitude of risk and degree of confidence in that assessment, based on study quality, effect sizes, biological plausibility, and consistency of Results Consensus was achieved by discussion and agreed at a multidisciplinary workshop.

Results From 16109 records, we included 69 papers (29 cohort, 4 case-control, 36 cross-sectional studies) providing data on 32 potential factors associated with an increased risk of asthma attacks. The panel had high confidence that previous asthma attacks were associated with greatly increased risk of future attacks. Poor access to care and persistent symptoms were associated with moderately/greatly increased risk. A moderately increased risk of attack was associated with suboptimal drug regimen (low controller/total therapy ratio), comorbid atopic/allergic disease, African-American ethnicity (US studies), poverty, and vitamin D deficiency. Environmental tobacco smoke (ETS) exposure, younger age, and obesity were associated with slightly increased risk. Gender, urban residence, and Hispanic ethnicity (US studies) were not associated with risk. The evidence for other factors was inconclusive.

Conclusions Assessment of clinical and demographic features (especially persistent symptoms, previous attacks, and sub-optimal drug regimen) may help clinicians to 'spot the child' at increased risk of asthma attacks and focus appropriate management. Population level factors (poverty, poor access to care) may be used by health service planners and policymakers to target healthcare initiatives.

\section{S63 DO THE ROYAL COLLEGE OF PHYSICIANS 'THREE QUESTIONS' PREDICT SYMPTOM CONTROL IN PAEDIATRIC ASTHMA?}

${ }^{1} \mathrm{G}$ Andrews, ${ }^{2} \mathrm{D}$ LO, ${ }^{2} \mathrm{M}$ Richardson, ${ }^{2} \mathrm{E}$ Gaillard. ${ }^{1}$ University of Leicester, Leicester, UK; ${ }^{2}$ Institute for Lung Health, NIHR Leicester Biomedical Research Centre and Department of Infection Immunity and Inflammation, University of Leicester, Leicester, UK

\subsection{6/thoraxjnl-2017-210983.69}

Introduction and Objectives The UK Quality Outcomes Framework (QOF) rewards primary-care practices for completing the Royal College of Physicians “Three Questions” (RCP3Q) score for all patients listed on their asthma register. Almost no validation data currently exists, however, to support its use in children. This study aimed to investigate the performance of the RCP3Q to predict asthma control in children, by comparing it with the validated Asthma Control Test (ACT) or Childhood Asthma Control Test (C-ACT).

Methods This was a prospective, observational study involving 8 primary-care practices. Children aged 5-16 on the QOF asthma register and/or receiving asthma medication were invited to self-complete the ACT (age 12-16, n=96) or CACT (age 5-11, n=223) questionnaire immediately prior to a primary-care asthma review, where responses to the RCP3Q were collected. RCP3Q scores were compared with ACT or C-ACT data to assess performance of the RCP3Q in predicting asthma control. The RCP3Q scoring system is summarised in figure 1.

Results Questionnaire and RCP3Q data was completed for 319 participants. RCP3Q scores correlated moderately with CACT and ACT data (Spearman's rho -0.49 and -0.52 respectively, $\mathrm{p}<0.001)$. A RCP3Q score of $\geq 2$ predicted uncontrolled asthma (C-ACT or ACT $\leq 19$ ) with a sensitivity of $57 \%$ and specificity of $81 \%$. A lower threshold RCP3Q score of $\geq 1$ gave a specificity of $55 \%$, resulting in a high false positive rate. A RCP3Q score of 0 predicted well-controlled asthma (C-ACT or ACT $\geq 20$ ) with a sensitivity of $55 \%$ and specificity of $81 \%$. Using thresholds of $\mathrm{RCP} 3 \mathrm{Q} \geq 2$ for uncontrolled asthma and $\mathrm{RCP} 3 \mathrm{Q}=0$ for good control resulted in $25 \%$ participants unclassified $(\mathrm{RCP} 3 \mathrm{Q}=1)$ and $18 \%$ of participants scoring 0,2 or 3 incorrectly classified. Binary logistic regression showed that individual positive answers to RCP questions 1 and 2, but not 3, significantly increased the likelihood of uncontrolled asthma.

Conclusions Our data in $\geq 300$ participants does not support use of the RCP3Q to classify asthma control in children. Our findings support current BTS/SIGN guidelines, which recommend use of validated asthma control questionnaires, such as C-ACT, when conducting a paediatric asthma review.

\section{Asthma: infection and inflammation}

\section{S64 CLINICAL AND TRANSCRIPTOMIC PROFILES OF SEVERE ASTHMATICS WITH HIGH OR LOW EXPRESSION OF THE GLUCOCORTICOID RECEPTOR AND IMPORTIN-7}

R Mullegama, S Pavlidis, KF Chung, IM Adcock, PK Bhavsar. Imperial College London, London, UK

\subsection{6/thoraxjnl-2017-210983.70}

Introduction and Objectives The majority of asthmatics can be well-controlled using inhaled corticosteroids (ICS) and longacting 32 -agonists (LABAs). However, approximately 5\% have a severe, refractory form of the disease and are often difficult to treat. Corticosteroid (CS) Insensitivity is the defining feature of these asthmatics known as "severe" asthmatics. The

In the last month:

1. Have you had difficulty sleeping because of your asthma symptoms (including cough)?

2. Have you had your usual asthma symptoms during the day (cough, wheeze, chest tightness or breathlessness)?

3. Has your asthma interfered with your usual activities (e.g housework, work, school etc)?

Score 1 for each yes answer, with total score ranging from 0 and 3 . 